\title{
Efficiency and safety of subconjunctival injection of anti-VEGF agent - bevacizumab - in treating dry eye
}

\section{Xiaodan Jiang* \\ Huibin Lv* \\ Weiqiang Qiu \\ Ziyuan Liu \\ Xuemin Li \\ Wei Wang}

Department of Ophthalmology, Peking University Third Hospital, Beijing,

People's Republic of China

*These authors contributed equally as first authors
Correspondence: Xuemin $\mathrm{Li}$ Department of Ophthalmology, Peking University Third Hospital, No 49 Huayuan North Road, Haidian District, Beijing 10019I, People's Republic of China

Tel +861082266312

Fax +86 I0 8208995 I

Email lxmlxm66@sina.com
This article was published in the following Dove Press journal:

Drug Design, Development and Therapy

12 June 2015

Number of times this article has been viewed

Purpose: Dry eye is a chronic inflammatory ocular surface disease with high prevalence. The current therapies for dry eye remain to be unspecific and notcomprehensive. This study aims to explore safety and efficacy of a novel treatment - subconjunctival injection of bevacizumab - in dry eye patients.

Methods: Sixty-four eyes of 32 dry eye patients received subconjunctival injection of $100 \mu \mathrm{L}$ $25 \mathrm{mg} / \mathrm{mL}$ bevacizumab. Dry eye symptoms, signs (corrected visual acuity, intraocular pressure, conjunctival vascularity, corneal staining, tear break-up time, Marx line score, and blood pressure), and conjunctival impression cytology were evaluated 3 days before and 1 week, 1 month, and 3 months after injection.

Results: Significant improvements were observed in dry eye symptoms, tear break-up time, and conjunctival vascularization area at all the visits after injection compared to the baseline $(P<0.05)$. The density of the goblet cell increased significantly at 1 month and 3 months after injection $(P<0.05)$. There was no visual and systemic threat observed in any patient.

Conclusion: Subconjunctival injection of $100 \mu \mathrm{L} 25 \mathrm{mg} / \mathrm{mL}$ bevacizumab is a safe and efficient treatment for ocular surface inflammation of dry eye disease.

Keywords: anti-VEGF, bevacizumab, dry eye, ocular surface inflammation, subconjunctival

\section{Introduction}

Dry eye is a chronic inflammatory ocular surface disease caused by a lot of factors, which is accompanied by increased osmolarity and instability of the tear film. ${ }^{1}$ Its main manifestations fall into ocular surface discomfort, intermittent blurred vision, and other potential ocular surface damages. ${ }^{2}$ The core mechanism of dry eye mainly begins with the low lacrimal flow or high evaporation driven by lots of factors and diseases, which results in the state of tear hyperosmolarity. ${ }^{3}$ Such hyperosmolarity causes damage to the surface epithelium by activating a cascade of inflammatory events at the ocular surface and releasing inflammatory mediators into the tears, such as IL-1 $\alpha$, IL-1 $\beta$, TNF- $\alpha$, MMP-9, vascular endothelial growth factor (VEGF), and epidermal growth factor. ${ }^{4}$ Epithelial damage involves cell death by apoptosis, a loss of goblet cells, and disturbance of mucin expression, leading to tear film instability. ${ }^{5}$ This instability exacerbates ocular surface hyperosmolarity and completes the vicious spiral of the dry eye mechanism. The crux of the treatment is to break down the vicious inflammatory spiral.

VEGF, also known as vascular permeability factor, plays an important role in angiogenesis. ${ }^{6}$ Moreover, it also exerts a very important role in wound healing, embryonic development, some tumor growths, and ascites formation. ${ }^{6}$ In the human 
body, there are two kinds of high-affinity receptors of VEGF: Flt-1 (VEGFR-1) and KDR (VEGFR-2). ${ }^{6}$ Previous studies showed that VEGF levels were increased in tear samples from patients with ocular chronic inflammatory diseases, such as vernal keratoconjunctivitis and dry eye. ${ }^{7}$ Yoo et $\mathrm{al}^{8}$ demonstrated that VEGF, acting as a direct proinflammatory factor, was able to activate endothelial cells by producing chemotactic factors, such as MCP-1 and IL-8. At the same time, VEGF can promote monocytes to release of proinflammatory factors, such as TNF- $\alpha$ and IL-6, which boost the process of inflammation. ${ }^{8}$ Angiogenesis and inflammation are two pathological processes that interact with each other, and VEGF is the key factor that promotes the crosstalk between angiogenesis and inflammation. ${ }^{9}$ Bevacizumab (Avastin; Genentech, South San Francisco, CA, USA) is a humanized monoclonal antibody, first approved by the US Food and Drug Administration to treat angiogenesis. It can bind to and inhibit the biologic activity of all kinds of VEGF subtypes of in vitro and in vivo assay systems. ${ }^{10}$ The present study aims to evaluate the efficacy and safety of subconjunctival injection of anti-VEGF - bevacizumab - in the dry eye patients.

\section{Materials and methods}

\section{Patients}

The present study was conducted according to the principles of the Declaration of Helsinki and was approved by the Human Research and Ethics Committee of Peking University Third Hospital. The written informed consent form was obtained from each participant before their enrollment.

Subjects were recruited from the outpatient department of the Department of Ophthalmology of Peking University Third Hospital between April 2013 and December 2013. The inclusion criteria for this study were 1 ) age $18-80$ years; 2) chronic symptoms of burning, foreign body sensation, or itching of both eyes for $>12$ months; 3) Schirmer I test $<5 \mathrm{~mm} / 5 \mathrm{~min}$ (without anesthesia) or breakup time $\leq 10$ seconds; ${ }^{11}$ and 4) willingness to cooperate with the doctors in the follow-up visits. Exclusion criteria included patients with 1) any structural abnormalities (such as eyelid trichiasis, entropion, and scarring); 2) the presence of pinguecula or pterygium; 3 ) active allergy or infection or inflammatory disease at the ocular surface unrelated to dry eye; 4) any inflammation or active structural change in the iris or anterior chamber; 5) glaucoma; 6) diabetes or other systemic, dermatologic, or neurologic diseases that affect the health of ocular surface; 7) use of any other topical medication other than artificial tears within the past month; 8) use of any systemic antiinflammatory drugs or medication that may interfere with tear production, such as antianxiety, antidepressive, and antihistamine medications within 3 months; 9) previous punctal occlusion or eye surgery; 10) a positive history of refractive surgery or contact lens wear; 11) hypertension that is unstable; and 12) a history of any bevacizumab contraindications, such as heart failure, gastrointestinal perforation, and pregnancy.

The study evaluated 64 eyes of 32 dry eye patients (13 males and 19 females). The mean age of the patients was $50.93 \pm 14.73$ years (range: $21-78$ years).

\section{Subconjunctival injection}

In the operating room, subjects received bilateral subconjunctival injection of bevacizumab $(100 \mu \mathrm{L}, 2.5 \mathrm{mg} / 0.1 \mathrm{~mL}$, Avastin; Genentech) with a $29 \mathrm{G}$ needle (1 mL; Becton, Dickinson and Company, Franklin Lakes, NJ, USA). Before the injection, a drop of ropivacaine hydrochloride (Benoxil; Santen Pharmaceutical Co., Ltd., Osaka, Japan) was instilled in the conjunctival sac to serve as a topical anesthetic. All the injection sites were identical, which were $3 \mathrm{~mm}$ beneath the inferior rim of cornea. A drop of levofloxacin eye drop (Ofloxacin; Santen Pharmaceutical Co., Ltd.) was applied to the eye immediately after the injection. All the injections were performed by the same doctor (WQ).

\section{Clinical evaluation}

Subjects were evaluated at five visits: 3 days before the injection and 1 day, 1 week, 1 month, and 3 months after the injection. The clinical assessments of the subject and both eyes were carried out in the following order: dry eye symptoms, blood pressure, visual acuity, intraocular pressure (IOP), bulbar conjunctival vascularization, conjunctival impression cytology, tear break-up time (TBUT), Marx line (ML) assessment, and corneal staining. At the day 1 visit after injection, only blood pressure, visual acuity, IOP, and slit lamp examination were required to assure the safety of the patients. An interval of 10 minutes was required between different tests. All the test data were collected by two doctors (JXD and LZY); the average would be defined as the final result. All the samples for the conjunctival impression cytology and their staining were performed by the same doctor (LHB) who was masked to the protocol.

\section{Symptom evaluation}

The Ocular Surface Disease Index (OSDI; Allergan, Inc., Irvine, CA, USA), which includes 12 questions, was used to assess the dry eye symptoms. Total OSDI score was 
calculated as follows: OSDI $=$ (sum of scores of all answered questions) $\times 25 /$ (total number of answered questions). Therefore, OSDI score ranges from 0 to 100 . The OSDI score of 0 indicates no symptoms, 100 means extremely serious, 12 is usually considered as the abnormal line, 13-22 means mild dry eye, 23-32 indicates moderate dry eye, and $\geq 33$ represents severe dry eye. ${ }^{12}$

\section{Conjunctival vascularization evaluation}

Conjunctival vascularization pictures were obtained with an IM 900 (Imaging module 900; Switzerland) slit lamp at a magnification of $16 \mathrm{x}$ by the same doctor (JXD). The pictures were analyzed using the ImageJ software program (v1.37). All the analysis was performed on ImageJ at the end of the follow-up. All measurements and analysis were performed by one person who was masked to the study protocol. According to the ImageJ analysis method from Bayar et al, ${ }^{13}$ the pictures were turned to black and white graphs and the proportion of bulbar conjunctival vascularization was measured in terms of pixels of bulbar conjunctival vessels divided by the pixels of the bulbar conjunctiva.

\section{Conjunctival cytology impression}

Cytologic specimens were obtained from temporal bulbar conjunctiva, $3 \mathrm{~mm}$ away from corneal limbus. Before the impression, a drop instillation of ropivacaine hydrochloride (Benoxil; Santen Pharmaceutical Co., Ltd.) was applied to the conjunctival sac to serve as a topical anesthetic. Cellulose acetate strip (Tianjin Jingming New Technological Development Co., Ltd, Tianjin, People's Republic of China) was firmly pressed against the sampled area for 20 seconds. The strip was immediately transferred into $95 \%$ alcohol and stained with periodic acid-Schiff. ${ }^{14}$ Cytology impression was performed at three visits, which were 3 days before and 1 month and 3 months after the injection.

\section{TBUT and corneal staining}

A total of 5 ( $\mu \mathrm{L}$ of $2 \%$ sodium fluorescein was instilled onto the bulbar conjunctiva without inducing reflex tearing, by using a micro-pipette. ${ }^{11}$ The patient was asked to blink naturally without squeezing for three to five times, and then the patient was asked to stare straight ahead without blinking, until told otherwise, under the cobalt blue light. ${ }^{11}$ A stopwatch was used to record the time between the last complete blink and the first appearance of a dry spot or disruption in the tear film. ${ }^{11}$ The procedure was evaluated for three times, and the average value was defined as the final score. For corneal staining evaluation, the cornea was divided into five sectors; ${ }^{15}$ each sector was graded from 0 to 3 using the following criteria: $0=$ no staining; $1=$ punctate/ stipple staining; $2=$ ball and linear staining; and 3= coalesced staining. ${ }^{16}$

\section{Meibomian gland function evaluation}

ML score was used to evaluate the meibomian gland function. ${ }^{17} \mathrm{~A}$ total of $5 \mu \mathrm{L}$ of $2 \%$ sodium fluorescein was instilled on the bulbar conjunctiva, and the patient was instructed to blink naturally without squeezing. The ML was assessed under the cobalt blue light of the slit lamp.

\section{Statistical analysis}

Statistical analysis was performed by using $\mathrm{R}$ software (Version 2.14.2). Comparison between data points was performed with an analysis of variance with Dunnett's post hoc test, which compared the symptoms and clinical grading and the percentage of bulbar conjunctival vascularization at the three different follow-up times to those at the baseline. Paired $t$-test was used to compare the goblet cell density at the two different follow-up times to that of the baseline.

We certify that all applicable institutional and governmental regulations concerning the ethical use of human volunteers were followed during this research.

\section{Results \\ Clinical symptoms and signs}

The results of OSDI score, evaluation of clinical signs, and the percentage of bulbar conjunctival vascularization are listed in Table 1.

According to the OSDI questionnaire, a significant decrease occurred at the time of 1 week, 1 month, and 3 months after injection compared to that of the baseline $(P<0.01)$. TBUT at the time of 1 week, 1 month, and 3 months after injection significantly increased compared to that at the baseline $(P<0.01)$. However, there was no significant change in the evaluation of corneal staining score or ML score during all the visits.

After ImageJ analysis of the percentage of bulbar conjunctival vascularization (Figure 1), a significant decrease was observed at 1 week, 1 month, and 3 months after injection compared to that of the baseline $(P<0.01)$. In the light of conjunctival cytology impression analysis (Figure 2), the goblet cell density was significantly increased at 1 month 
Table I OSDI scores, clinical grading scores, and percentage of bulbar conjunctival vascularization area in the treatment (subconjunctival bevacizumab) of eyes at all visits compared with the baseline

\begin{tabular}{|c|c|c|c|c|}
\hline \multirow[t]{2}{*}{ Symptoms and signs } & \multirow{2}{*}{$\begin{array}{l}\text { Baseline } \\
\text { (minimum-maximum) }\end{array}$} & \multicolumn{3}{|c|}{ Median (minimum-maximum) } \\
\hline & & I wk & I mo & $3 \mathrm{mos}$ \\
\hline OSDI & $31.82(0.00-100.00)$ & $18.18(0.00-75.00)$ & $16.67(0.00-64.42)$ & $19.00(0.00-50.00)$ \\
\hline$p^{*}$ & & $0.00 I^{* *}$ & $<0.00 I^{* *}$ & $0.002 * *$ \\
\hline TBUT & $4.0(0.0-11.0)$ & $5.5(1.0-14.0)$ & $6.5(3.0-20.0)$ & $6.0(2.0-20.0)$ \\
\hline$p^{*}$ & & $<0.00 I^{* *}$ & $<0.00 I^{* *}$ & $<0.00 I^{* *}$ \\
\hline Corneal staining & $0.0(0.0-9.0)$ & $0.0(0.0-8.0)$ & $0.0(0.0-6.0)$ & $0.0(0.0-6.0)$ \\
\hline$P^{*}$ & & 0.0644 & 0.192 & 0.209 \\
\hline Marx line & $3.5(0.0-9.0)$ & $3.5(0.0-9.0)$ & $2.0(0.0-9.0)$ & $2.0(0.0-9.0)$ \\
\hline$p^{*}$ & & 0.354 & 0.459 & 0.69 \\
\hline Conjunctival vascularization area & $0.139(0.085-0.256)$ & $0.125(0.065-0.202)$ & $0.113(0.070-0.187)$ & $0.105(0.079-0.164)$ \\
\hline$P^{*}$ & & $<0.00 I^{* *}$ & $<0.00 I^{* *}$ & $0.005^{* *}$ \\
\hline
\end{tabular}

Notes: *Dunnett test; $* * P<0.05$.

Abbreviations: OSDI, Ocular Surface Disease Index; wk, week; mo, month; TBUT, tear break-up time.

and 3 months after injection compared to that of the baseline $(P<0.05)$ (Table 2).

\section{Safety data}

During the study, in all subjects, corrected visual acuity was not decreased. IOP was lower than $21 \mathrm{mmHg}$, and the blood pressure was beneath 140/90 $\mathrm{mmHg}$. Ten eyes of nine subjects were identified with subconjunctival hemorrhage 1 day after injection. A weekly follow-up was conducted for these patients, and all hemorrhage was absorbed within 2 weeks. No systemic adverse event was observed during the study.

\section{Discussion}

Dry eye is a chronic inflammatory ocular surface disease. ${ }^{1}$ According to the tear samples analysis from dry eye patients, the levels of numerous cytokines and chemokines increase dramatically, such as VEGF, epidermal growth factor, $\mathrm{CX}_{3} \mathrm{CL}_{1}$, IL-8/CXCL8, CXCL10, IL-6, and TNF- $\alpha .{ }^{18}$ Goyal et $\mathrm{al}^{19}$ first evaluated the effects of intraperitoneal injections of $400 \mu \mathrm{g}$ of anti-VEGF antibody in a murine model with dry eye and found that the ocular inflammation was significantly relieved after the injection, as well as the dry eye signs. To our knowledge, there is no study reporting the application of bevacizumab to dry eye patients in the clinic. Our study applied sunconjunctival injection of anti-VEGF therapy to the dry eye patients and aimed to assess its safety and efficacy. Dry eye symptoms and signs were significantly improved after subconjunctival injection of $100 \mu \mathrm{L} 25 \mathrm{mg} /$ $\mathrm{mL}$ bevacizumab in this study.

The OSDI questionnaire was used to assess the dry eye symptoms. Three contents are included in the OSDI questionnaire, which are ocular discomfort, visual function, and environmental triggers. ${ }^{14}$ For a long time, dry eye has been also considered as a chronic pain disease. ${ }^{16}$ Pain is a subjective phenomenon that varies greatly from person to person. Much research indicates that most of the proinflammatory factors (such as IL-1 $\beta$, TNF- $\alpha$, IL-6, and IL-8/ CXCL8) are able to lower the pain threshold and the antiinflammatory factors (such as IL-10) can reduce the ability to perceive pain perception. ${ }^{20}$ Studies show that VEGF plays an important role in the nervous system. Lin et $\mathrm{al}^{21}$ found that VEGF and VEGFR-2 were involved in the pathogenesis of neuropathic pain and VEGF inhibitors were effective for pain relief, which indicated that VEGF may be the key factor that promotes the crosstalk between vascular and nerve systems. The discomfort of dry eye may be due to the effects of cytokines such as VEGF on the nerve endings of conjunctiva and cornea, which results in hyperalgesia, yielding to foreign body sensation, eye pain, and so on. The present study applied subconjunctival injection of bevacizumab to dry eye patients, and a significant decrease in OSDI score was achieved, which means that the dry eye symptoms were significantly relieved. Our results were similar to those of other studies. Enkvetchakul et a ${ }^{22}$ used bevacizumab in the treatment of pterygium and found out that the ocular irritation symptoms were significantly improved.

Our study also found that TBUT was significantly lengthened after the injection of bevacizumab. Tear film is a highly organized structure on the ocular surface; its stability and function are highly dependent on its biochemical composition. ${ }^{23}$ Enríquez-de-Salamanca et al ${ }^{24}$ indicated that the expression of proinflammatory factors was negatively correlated with TBUT. Marsovszky et $\mathrm{al}^{25}$ also demonstrated that active inflammation of the ocular surface of the patients with rheumatoid arthritis could decrease the TBUT level. In our research, the longer TBUT after the injection may be the result of the inhibition effect of the anti-VEGF therapy on the expression and activation 
A

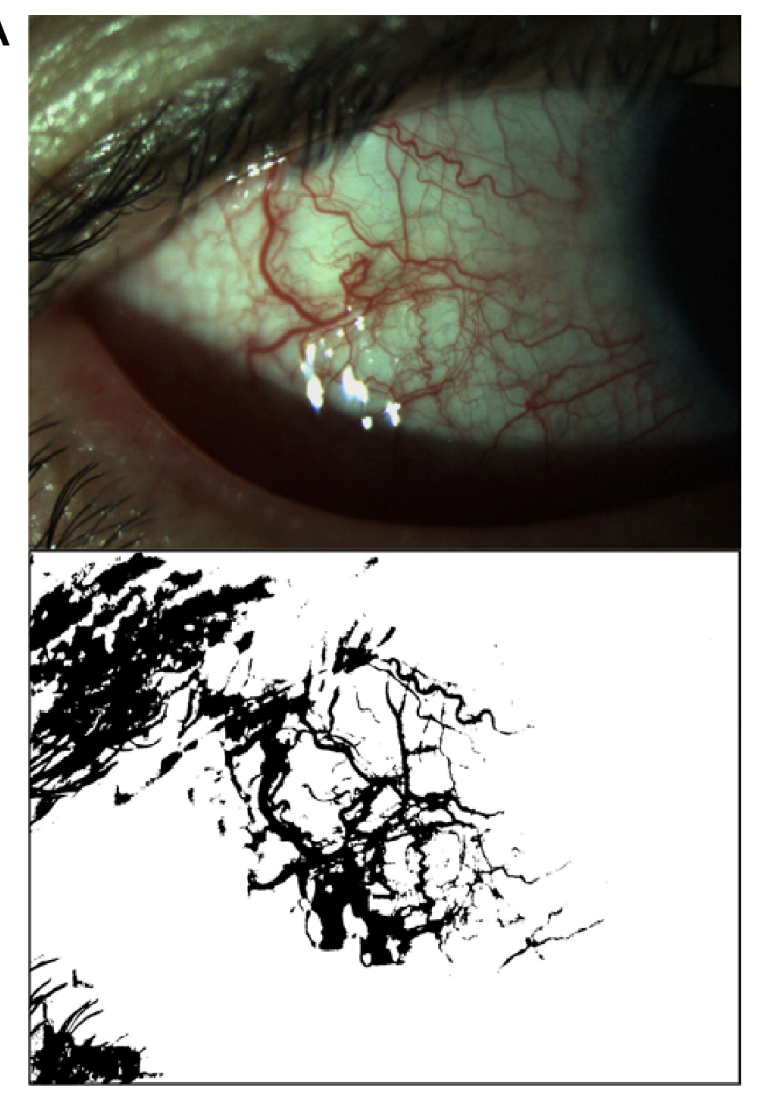

C

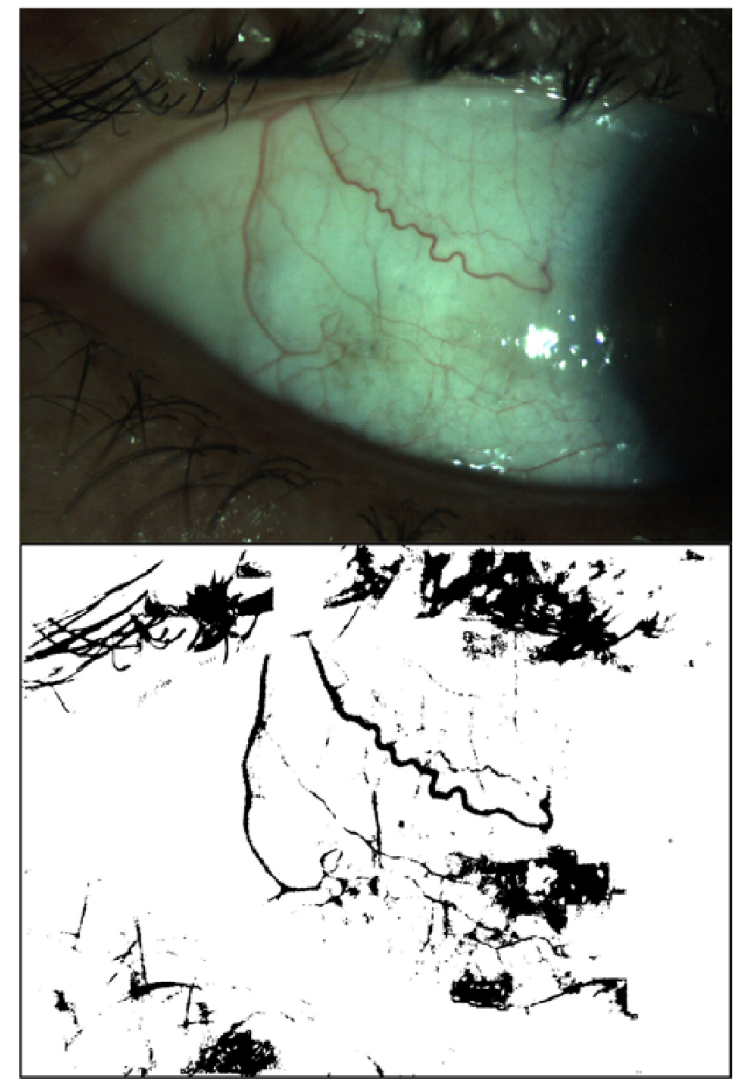

B
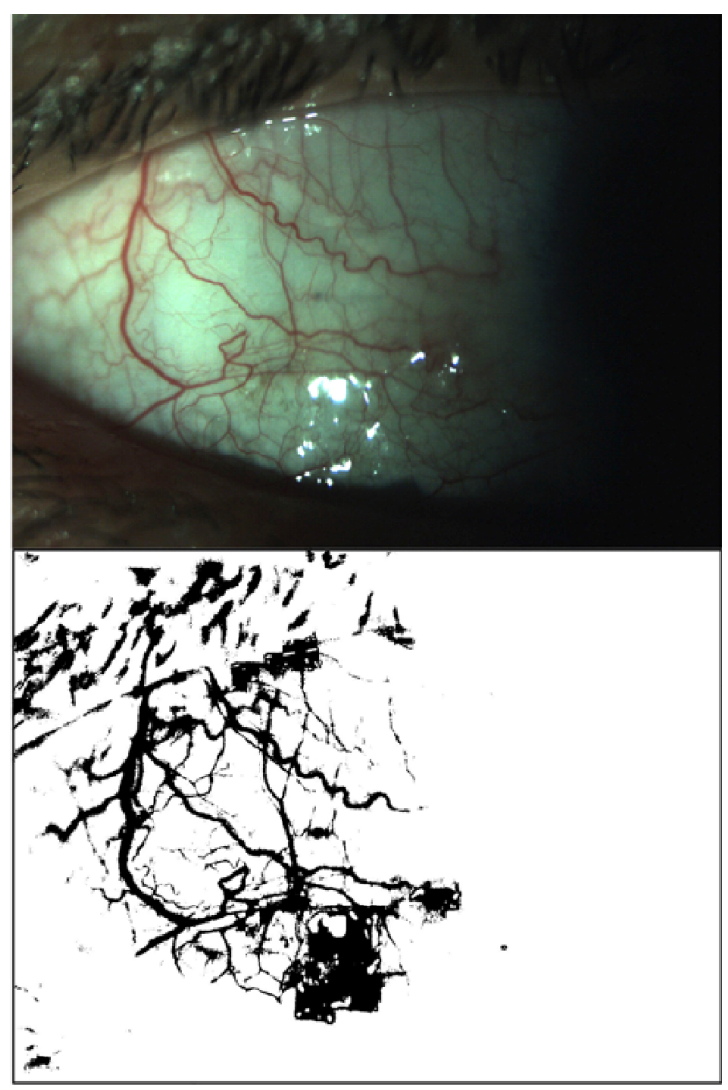

D

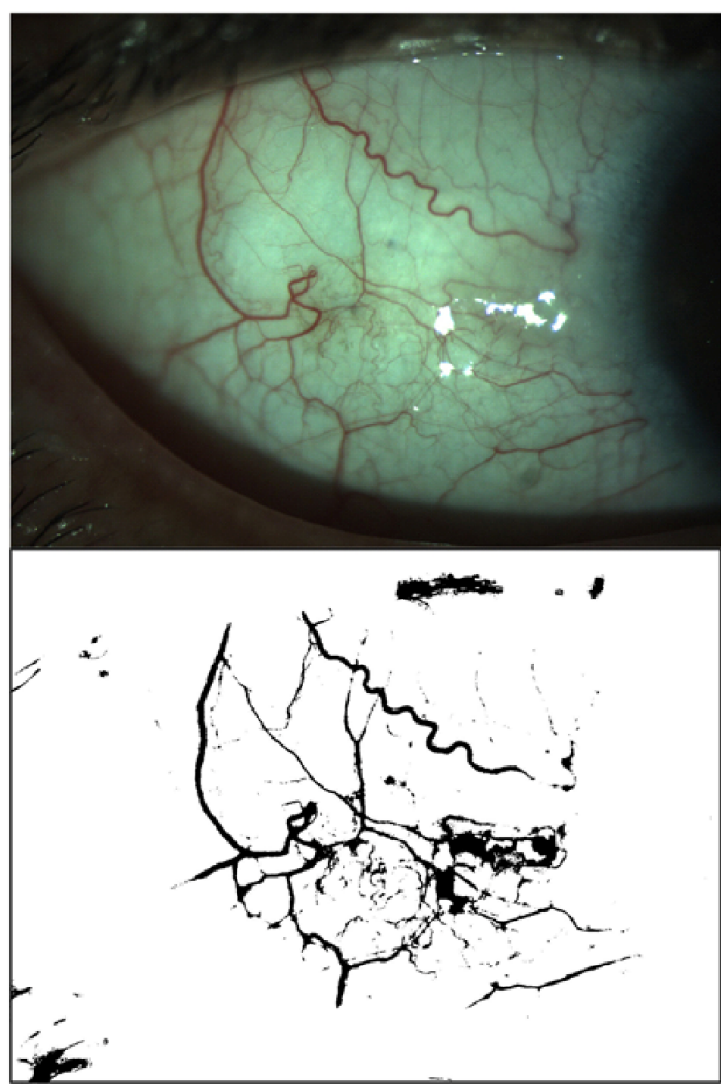

Figure I Bulbar conjunctival vascularization area.

Notes: The upper row images are the conjunctival vascularization pictures obtained with an IM900 slit lamp. The lower row images are the correspondent vascular pictures analyzed with Image J software. (A) is at 3 days before injection. High vascular density with a wide diameter could be observed. (B) refers to I week after injection. (C) is at I month after injection. (D) is at 3 months after injection. The vasculars in (B-D) are much thinner in diameter with lower density. All the dark pixels resulted from the light reflection were excluded from calculation. The pixels of the eyelid and eyelash were excluded. 


\section{A}

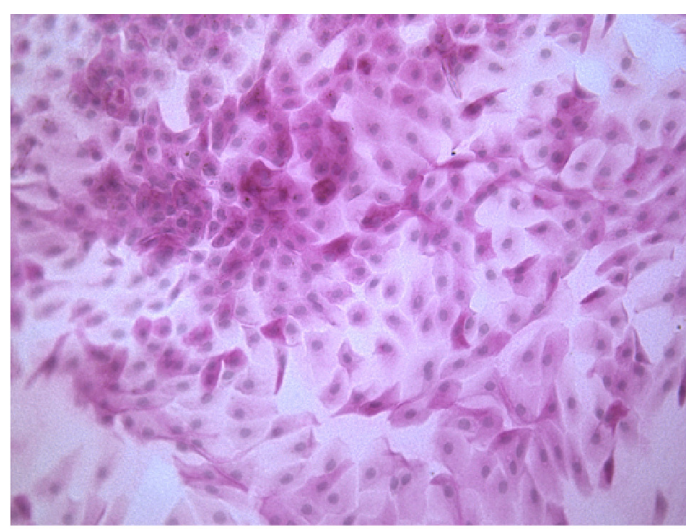

B

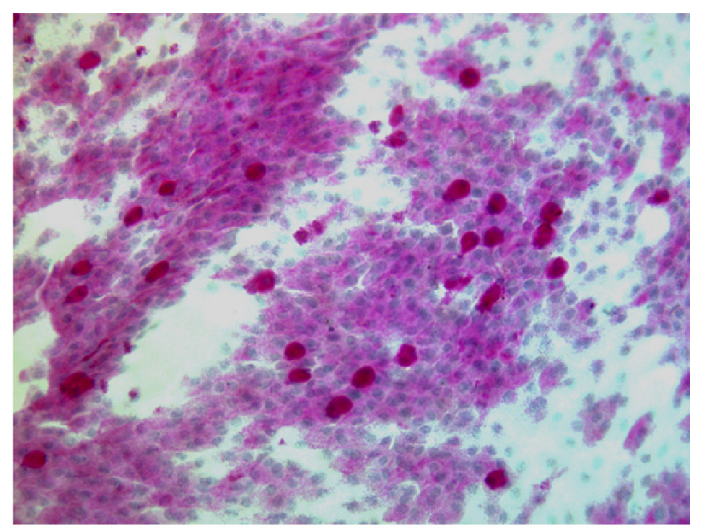

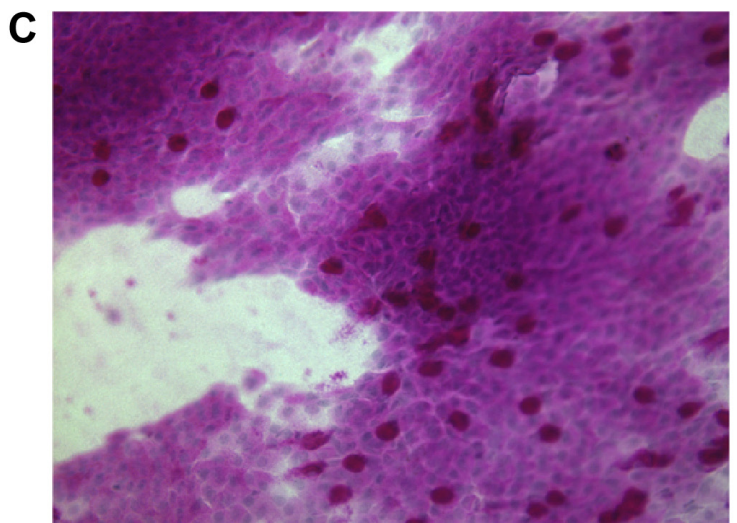

Figure 2 Conjunctival cytology analysis (stained with periodic acid-Schiff, 40x).

Notes: (A) refers to the staining before injection. (B) represents the staining I month after injection. (C) is the staining 3 months after injection. Compared to (A), the epithelial in (B) and (C) is more regular, rounder and smaller, the cell gap in (B) and (C) is much closer. The density of goblet cell, is much higher in (B) and (C), compared to $(\mathbf{A})$.

of the proinflammatory factors, thereby depressing the ocular surface inflammation.

Our results showed no significant change in corneal staining after the anti-VEGF therapy, which is inconsistent with the findings of Goyal et al ${ }^{19}$ who found that after intraperitoneal injections of anti-VEGF antibody in a murine model with dry eye, corneal staining significantly improved. This conflict may be due to the differences in the study subjects. Our study first applied anti-VEGF to the dry eye patients, $69 \%$ of whom had no corneal damage. This may be the reason why there are no significant differences. According to

Table 2 Conjunctival goblet cell density in the treatment (subconjunctival bevacizumab) of eyes at I mo and 3 mos after injection compared with the baseline

\begin{tabular}{llll}
\hline \multirow{4}{l}{ Average \pm SD $\left(\mathbf{m m}^{2}\right)$} \\
\cline { 2 - 4 } & Baseline & $\mathbf{I}$ mo & $\mathbf{3}$ mos \\
\hline Goblet cell density & $269.44 \pm I 04.20$ & $308.23 \pm 84.29$ & $323.67 \pm \mathrm{II} 2.23$ \\
$P^{*}$ & & $0.018^{* *}$ & $0.028^{* *}$ \\
\hline
\end{tabular}

Notes: $*$ Paired $t$-test; $* *<0.05$.

Abbreviations: mo, month; SD, standard deviation. the corneal staining analysis of some patients (Figure 3), the anti-VEGF was indeed able to relieve the corneal damage. This improvement may be the result of a better tear film or the relieving of ocular surface inflammation, which makes the corneal epithelial injury restoration possible. Our results, on the other hand, indicated the nontoxicity of this therapy to the corneal epithelium.

According to conjunctival cytology impression analysis, a significant increase in the goblet cell density was observed after the injection of bevacizumab. Contreras-Ruiz et $\mathrm{al}^{26}$ indicated that the apoptosis of goblet cells was primarily induced by TNF- $\alpha$ and IFN- $\gamma$. VEGF is able to promote the expression of TNF- $\alpha$. So, the increase in the goblet cell density may be due to the improved ocular surface inflammation after the therapy, which improved the microenvironment of goblet cells as well as the cells' ability to self-heal.

However, Joussen et $\mathrm{al}^{27}$ found out that in a murine model of limbal insufficiency, VEGFR was abundant in the corneal goblet cells and the systemic inhibition of VEGF bioactivity potently suppressed the corneal goblet cell density, which disagreed with our results. The inconsistency may be due to 

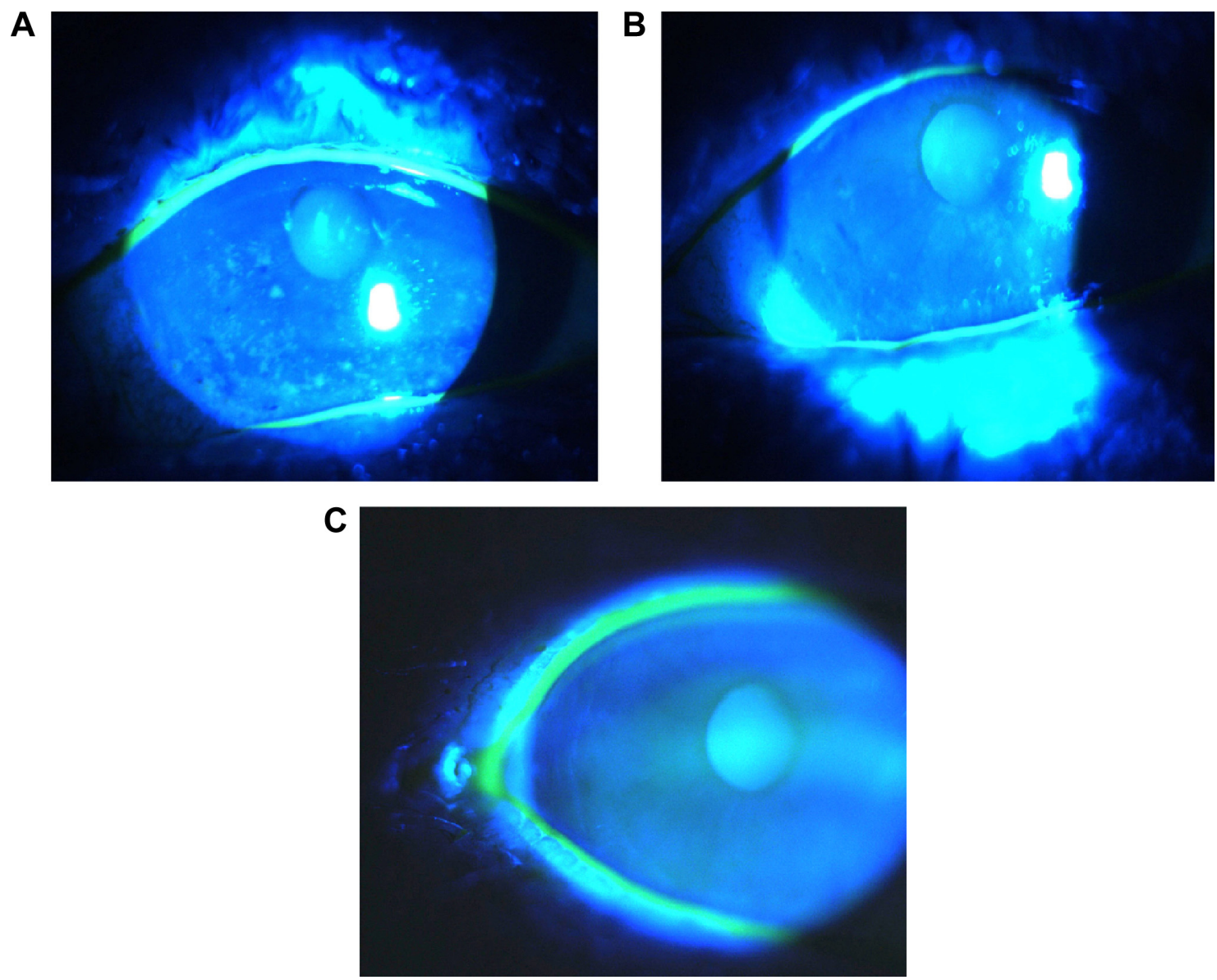

Figure 3 Corneal staining pictures

Notes: (A) refers to the staining picture obtained before injection. (B) represents the staining picture obtained I month after injection. (C) is the staining picture taken 3 months after injection. A dramatic improvement in corneal staining could be observed in (B) and (C), compared to (A).

two reasons. One is that the goblet cell studied by Joussen was pathologically growing on the cornea, which was different from that grown in the conjunctiva. The other reason may be due to the improvement of the microenvironment of the goblet cell exceeding the direct adverse effect that antiVEGF therapy played on the goblet cell. Therefore, further research is needed to confirm our hypotheses.

There are some possible mechanisms whereby anti-VEGF could relieve the dry eye inflammation. First, it decreases the permeability of the conjunctival vascular membrane, thus reducing the leakage of cytokines and leukocytes to the ocular surface. ${ }^{28}$ Second, it reduces the vascular endothelial area, thereby inhibiting the adhesion and migration of the leukocytes. ${ }^{9}$ Third, it inhibits the production of cytokines and chemokines from the endothelial cells. ${ }^{9}$ Fourth, it reduces cytokine production from the VEGF-induced monocytes or macrophages. ${ }^{10}$ Finally, it suppresses the nerve irritation from VEGF or other cytokines, thus relieving the hyperalgesia. These mechanisms may happen independently, but it remains to be confirmed which mechanism has a principal impact on the quenching of dry eye inflammation.

The limitations of our study include: the short time of observation that was limited to 3 months, the small sample size, and the lack of control group which cannnot then rule out the effect of the subconjunctival injection procedure. Further research is needed to be carried out to ascertain the doses of the injection and the long-term safety and efficacy of anti-VEGF therapy, as well as the influence of the subconjunctival injection procedure on the results.

\section{Conclusion}

Dry eye disease is a chronic inflammatory disease characterized by a cascade of inflammatory events at the ocular surface. The treatments of dry eye, which are manifold, remain inefficient and notcomprehensive. Our study applied anti-VEGF agent - bevacizumab - to the eyes of dry eye patients and demonstrated that it was able to relieve the symptoms and signs of dry eye safely and effectively for a 
period up to 3 months after therapy, which may open up a potential new treatment for dry eye.

\section{Acknowledgments}

The authors acknowledge Dr Ruo Lin for her great help in manuscript writing.

\section{Disclosure}

The authors report no conflicts of interest in this work. The authors alone are responsible for the content and writing of the paper.

\section{References}

1. Massingale ML, Li X, Vallabhajosyula M, Chen D, Wei Y, Asbell PA. Analysis of inflammatory cytokines in the tears of dry eye patients. Cornea. 2009;28:1023-1027.

2. Calonge M, Enríquez-de-Salamanca A, Diebold Y, et al. Dry eye disease as an inflammatory disorder. Ocul Immunol Inflamm. 2010;18(4):244-253.

3. Bron AJ, Tiffany JM, Yokoi N, Gouveia SM. Using osmolarity to diagnose dry eye: a compartmental hypothesis and review of our assumptions. Adv Exp Med Biol. 2002;506:1087-1095.

4. Li DQ, Chen Z, Song XJ, Luo L, Pflugfelder SC. Stimulation of matrix metalloproteinases by hyperosmolarity via a JNK pathway in human corneal epithelial cells. Invest Ophthalmol Vis Sci. 2004;45:4302-4311.

5. Yeh S, Song XJ, Farley W, Li DQ, Stern ME, Pflugfelder SC. Apoptosis of ocular surface cells in experimentally induced dry eye. Invest Ophthalmol Vis Sci. 2003;44:124-129.

6. Semeraro F, Morescalchi F, Duse S, Parmeggiani F, Gambicorti E, Costagliola C. Aflibercept in wet AMD: specific role and optimal use. Drug Des Devel Ther. 2013;7:711-722.

7. Abu El-Asrar AM, Al-Mansouri S, Tabbara KF, Missotten L, Geboes K. Immunopathogenesis of conjunctival remodelling in vernal keratoconjunctivitis. Eye (Lond). 2006;20:71-79.

8. Yoo SA, Kwok SK, Kim WU. Proinflammatory role of vascular endothelial growth factor in the pathogenesis of rheumatoid arthritis: prospects for therapeutic intervention. Mediators Inflamm. 2008;2008:129873.

9. Ferrara N, Gerber H-P, LeCouter J. The biology of VEGF and its receptors. Nat Med. 2003;9:669-676.

10. Sandler A, Gray R, Perry MC, et al. Paclitaxel-carboplatin alone or with bevacizumab for non-small-cell lung cancer. $N$ Engl J Med. 2006;355:2542-2550.

11. Foulks GN. Report of the International Dry Eye WorkShop (DEWS). Ocul Surf. 2007;5:61-204.

12. Ozcura F, Aydin S, Helvaci MR. Ocular surface disease index for the diagnosis of dry eye syndrome. Ocul Immunol Inflamm. 2007;15(5):389-393.
13. Bayar SA, Kucukerdonmez C, Oner O, Akova YA. Subconjunctival bevacizumab in the impending recurrent pterygia. Int Ophthalmol. 2014;34:541-547.

14. Li XM, Hu L, Hu J, Wang W. Investigation of dry eye disease and analysis of the pathogenic factors in patients after cataractsurgery. Cornea. 2007;26:16-20.

15. Caffery BE, Josephson JE. Corneal staining after sequential instillations of fluorescein over 30 days. Optom Vis Sci. 1991;68:467-469.

16. Thomas ML, Szeto VR, Gan CM, Polse KA. Sequential staining: the effects of sodium fluorescein, osmolarity, and $\mathrm{pH}$ on human corneal epithelium. Optom Vis Sci. 1997;74:207-210.

17. Yamaguchi M, Kutsuna M, Uno T, Zheng X, Kodama T, Ohashi Y. MarX line: fluorescein staining line on the inner lid as indicator of meibomian gland function. Am J Ophthalmol. 2006;141:669-675.

18. Benito MJ, González-García MJ, Tesón M, et al. Intra-and inter-day variation of cytokines and chemokines in tears of healthy subjects. Exp Eye Res. 2014;120:43-49.

19. Goyal S, Chauhan SK, Dana R. Blockade of prolymphangiogenic vascular endothelial growth factor $\mathrm{C}$ in dry eye disease. Arch Ophthalmol. 2012;130:84-89.

20. Wang XM, Hamza M, Wu TX, Dionne RA. Upregulation of IL-6, IL-8 and CCL2 gene expression after acute inflammation: correlation to clinical pain. Pain. 2009;142:275-283.

21. Lin J, Li G, Den X, et al. VEGF and its receptor-2 involved in neuropathic pain transmission mediated by $\mathrm{P} 2 \mathrm{X}_{2}(/)_{3}$ receptor of primary sensory neurons. Brain Res Bull. 2010;83:284-291.

22. Enkvetchakul O, Thanathanee O, Rangsin R, Lekhanont K, SuwanApichon O. A randomized controlled trial of intralesional bevacizumab injection on primary pterygium: preliminaryresults. Cornea. 2011;30:1213-1218.

23. Puinhas A, Sampaio P, Castanheira EM, Real Oliveira ME, Lira M. Comparison of IgA, TNF- $\alpha$ and surface tension of the tear film in two different times of the day. Cont Lens Anterior Eye. 2013;36:140-145.

24. Enríquez-de-Salamanca A, Castellanos E, Stern ME, et al. Tear cytokine and chemokine analysis and clinical correlations in evaporative-type dry eye disease. Mol Vis. 2010;16:862-873.

25. Marsovszky L, Resch MD, Németh J, et al. In vivo confocal microscopic evaluation of corneal Langerhans cell density, and distribution and evaluation of dry eye in rheumatoid arthritis. Innate Immun. 2013;19:348-354.

26. Contreras-Ruiz L, Ghosh-Mitra A, Shatos MA, Dartt DA, Masli S. Modulation of conjunctival goblet cell function by inflammatory cytokines. Mediators Inflamm. 2013;2013:636812.

27. Joussen AM, Poulaki V, Mitsiades N, et al. VEGF-dependent conjunctivalization of the corneal surface. Invest Ophthalmol Vis Sci. 2003;44:117-123

28. Messenger WB, Beardsley RM, Flaxel CJ. Fluocinolone acetonide intravitreal implant for the treatment of diabetic macular edema. Drug Des Devel Ther. 2013;7:425-434.
Drug Design, Development and Therapy

\section{Publish your work in this journal}

Drug Design, Development and Therapy is an international, peerreviewed open-access journal that spans the spectrum of drug design and development through to clinical applications. Clinical outcomes, patient safety, and programs for the development and effective, safe, and sustained use of medicines are a feature of the journal, which

\section{Dovepress}

has also been accepted for indexing on PubMed Central. The manuscript management system is completely online and includes a very quick and fair peer-review system, which is all easy to use. Visit http://www.dovepress.com/testimonials.php to read real quotes from published authors. 